\title{
SIMULATION OF ADAPTIVE PROJECT MANAGEMENT ANALYTICS
}

\author{
Léa A. Deleris \\ Sugato Bagchi \\ Shubir Kapoor \\ Kaan Katircioglu \\ Richard Lam \\ Steve Buckley \\ IBM Research \\ Mathematical Sciences \\ TJ Watson Research Center \\ 1101 Kitchawan Road \\ Yorktown Heights, N.Y. 10598, U.S.A.
}

\begin{abstract}
Typically, IT projects are delivered over-budget and behind schedule. In this paper, we explore the effects of common project management practices that contribute to these problems and suggest a better alternative that can utilize resources more effectively. Our alternative approach uses (a) a thorough analysis of risks affecting activities in a project plan (i.e., the root factors leading to cost and time overruns), and (b) an optimization of the resources allocated to each activity in the project plan to maximize the probability of on time and within budget project completion. One key feature of our method is its capability to adapt and learn the risk factors affecting activities during the course of the project, enabling project managers to reallocate resources dynamically to ensure a better outcome given the updated risk profile. We use simulations to test the performance of our optimization algorithm and to gain insights into the benefits of adaptive re-planning.
\end{abstract}

\section{INTRODUCTION}

Project management is a critical competency in the services sector. It manages the activities necessary to perform and deliver a service to a customer. In this respect, it is analogous to the production process in the manufacturing sector and perhaps even more critical due to the presence of a customer during the 'production' of a service.

However, the importance of project management towards the successful performance of a service is not matched by the level of sophistication in the analytical tools available to a project manager and as such, some parts of this critical services competency remain more of an art than science. Consequently, it is not uncommon to see that services projects fail to deliver acceptable levels of customer satisfaction or do so behind schedule and/or over budget.

Typical project management tools that are available to project managers serve only to record a plan of activities and their expected resource allocations. Factors that might make the project deviate from the plan, such as risks that delay activities or the unavailability of resources to perform activities are not usually elaborately represented in such tools. This makes a quantitative evaluation of the project risk difficult. Risk factors are usually described in separate documents and without any specification of their impact on activity duration and cost. Even when the impact of a risk factor on activity durations is quantified, the correlation among these risk factors and their impacts (possibly mediated through common risk factors or root causes) are not modeled in the existing project management tools. Because of this limitation, deciding which risk factors to mitigate also has to occur outside the scope of the project plan.

Similarly, the measures available to get a delayed project back on schedule are also not explicitly modeled by the existing project management tools. Experienced project managers intentionally place slack in the plan to absorb delays and know which activity durations could be shortened by allocating additional or more skilled resources. Without the explicit representation of these resource alternatives, project management tools cannot aid resource allocation decisions.

In order to address these shortcomings, we have developed a project management model that explicitly includes risk factors and their impacts on activity costs and durations as well as alternative staffing scenarios associated with activities in a project plan. With this model, we 


\section{Deleris, Bagchi, Kapoor, Katircioglu, Lam and Buckley}

can perform risk assessment of project cost and time and incorporate this into the optimization of a project plan that is most robust against these risks (Deleris et al 2007).

The objective of this paper is to study the benefits of modeling risk factors and adaptive re-planning of a project in light of updated information about the risk factors and the progress of the project. We will use discrete event simulation to do this.

Section 2 provides the business context for the issues we wish to study in simulation. Section 3 presents a summary description of our risk and resource allocation analytics. Section 4 describes the simulation experiments using these analytics and explains the results.

\section{BUSINESS CONTEXT FOR SIMULATION}

We have chosen the domain of services transition projects for our experiments to evaluate the benefits of risk-based project plan generation and adaptive re-planning. A transition project is performed after a service provider has won an outsourcing contract with a client. It sets up the necessary business processes and IT systems in order to deliver the outsourced service to the client. Transition projects are notoriously prone to numerous risks of cost and time overruns. One of the key reasons for this is that transition happens between the "sell" and "deliver" phases of the service lifecycle and the business incentives in these two phases can be misaligned. Sales people who are rewarded for selling an engagement may make overly optimistic assumptions about resource needs of a transition project in order to increase their chances to win the engagement. As a result, people who are responsible for the successful delivery of a transition project may find it difficult to meet the level of commitment within budget. This requires a careful project planning and execution in both phases of the cycle. This planning must include the risk factors that are specific to the engagement and their potential impact on project delivery performance.

\subsection{Value of Risk-based Planning}

Based on a model of transition project risks, we wish to compare the performances of two different approaches to project planning. The first approach considers multiple risk scenarios and their impact on activity durations. It uses the best possible resource scenarios in order to minimize project cost subject to a target project duration. The second approach uses a project plan that does not take into account risks and does not allow the use of alternative resource scenarios. We are also interested in understanding how robust these project plans are. Here we define robustness as the ability to meet the target project deadline when the activity durations are subject to delays caused by a chosen risk scenario.
A significant difference in completion time between the two approaches will demonstrate the value of risk modeling and the resulting benefit of risk-based planning.

\subsection{Value of Periodic Re-planning}

The next issue to evaluate is the ability to re-plan periodically during the course of project execution. As activities are reported complete, their actual durations could be used to assess the progress of the project against the planned progress. Can periodic re-planning help to perform a 'course correction' on the project in light of delays that have already been incurred and help us remedy these delays? This is the basic question we would like to answer.

The added benefit of periodic re-planning may be minimal if the initial risk-based plan is robust in meeting the target project deadline across multiple risk scenarios. Even in this case, we wish to evaluate whether periodic replanning can achieve the deadline with a lower cost. In addition, can periodic re-planning allow us to achieve the deadline more accurately with a lower absolute error than the initial plan? This is hypothesized because the initial plan may seek to achieve robustness by completing ahead of schedule and thereby building up some slack in the project to hedge against risk.

\section{APRM METHOD}

\subsection{Literature Review}

A variety of papers catalog the risks in IT projects related to software development (see, for example, Taylor 2005). A recent paper (Taylor 2006) discussed risks in outsourcing engagements, agreeing that overoptimistic schedules and budgets are the most likely risks. Another source (Cole 1995) also identified these risk factors as significant in runaway IT projects.

Despite the amount of work in identifying risk factors, however, there is little agreement on how to quantitatively estimate or mitigate risks during project implementation. Approaches based on Bayesian Belief Networks (Pearl 1988) seem a natural fit to this problem. A procedure incorporating BBNs to support decision-making while continuously monitoring risks during project execution has been reported (Fan and $\mathrm{Yu}$ 2004). Another paper (Nasir 2003) captured the relationships among project risks using a BBN and used the output of the network to estimate the durations of categories of project activities. Our work is similar to this approach, but we tie specific risk elements to individual project activities, customizing the effects of the risk factors to each project plan. 


\section{Deleris, Bagchi, Kapoor, Katircioglu, Lam and Buckley}

\subsection{Risk Analysis}

We incorporate the notion of risk through the addition of a Bayesian Belief Network (BBN). This network captures how risk factors identified by project managers, such as "clarity of contract terms" or "resource availability", influence individual activity durations. Specifically, we start from a list of risk factors common across all outsourced services transition projects and structure them into a BBN. The conditional probability tables underlying the Bayesian Belief Network are estimated based on historical data about risk factors present in past projects, or if needed, elicited from experts. This corresponds to the upper part of Fig 1. Then, we seek expert opinion from project managers to link activities of a project to risk factors that are present and to specify the strength of the link. This provides the structure of the lower part of Fig. 1.

The risk network that we construct combines projectspecific information and information gathered from past projects. This enables several types of learning. First, the BBN enables learning during the course of the project from completed activity durations. The basic idea is that if early activities suffer from delays, it is likely that one or more risk factors are present. Thus, future activities will take more time than expected. While this idea is fairly intuitive, the interpolation of current delays towards future delays is less straightforward. There, the BBN structure automates the estimation, updating probabilities of risk factors present based on observed completion times and then updating the duration of future activities impacted by these risk factors. These computations are readily carried out through BBN software (e.g., the GeNIE software developed by the Decision Systems Laboratory of the University of Pittsburgh, 2007. Second, the top structure of the BBN, being common across projects, can be learned from historical data, and in turn updated after a project is completed. Several algorithms are available for this purpose (see, for instance, Heckerman, Geiger, and Chickering 1995 and Myers, Blackmond-Laskey, and Levitt 1999). Some algorithms even accommodate missing data. Note that standardization of project activities would enable learning the full network, including the links from risk factors to activity durations.

\subsection{Resource Allocation}

In this section we describe the algorithm designed to reduce project risk through an optimal allocation of resources across the project plan activities. To achieve this, we model a set of alternative resource scenarios that can be associated with an activity. Each resource scenario for a given activity is characterized by:

- A set of resources (i.e. skill types required to perform the activity),
- Number of resources (i.e. headcounts) required for each skill type,

- Experience level for each skill type, and

- Estimated activity duration (risk-less) for a given set of resources.

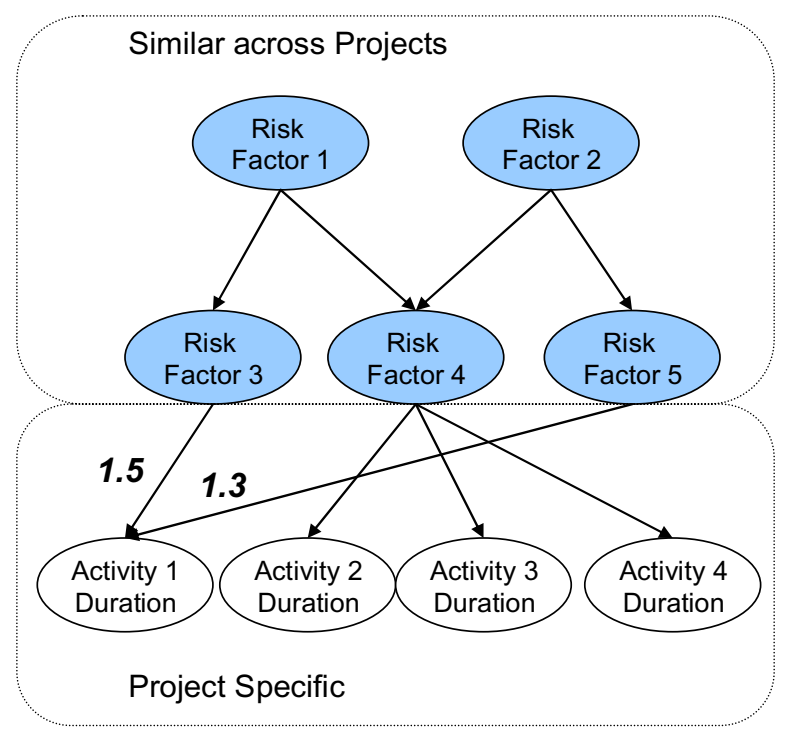

Figure 1: Illustrative Bayesian Network representing risk factors and their impacts on activity durations.

The decision variables of the optimization problem are the resource scenario selections for all activities. The objective function can be defined in a number of ways depending on the business focus. For instance, minimize expected project cost subject to expected project duration not exceeding a target.

To incorporate uncertainty from risk factors into the optimization, we use Monte Carlo simulation. Each repetition of the simulation is associated with one sample path of activity duration derived from the BBN, (i.e., a set of activity duration multipliers which are sampled from the BBN).

It is not possible in practice to evaluate all combinations of resource scenarios, as there are potentially too many combinations. For instance, a project with 100 activities, each having 5 resource scenarios, has $5^{100}$ potential combinations. Therefore, we designed a heuristic to prune scenarios that are not likely to be optimal. Its description can be found in Deleris et al.

The algorithm can be used in a number of different ways during project implementation. Based on the objective function defined, before the start of the project, it can be used to analyze the resource scenarios and find the minimum budget requirements that achieve project duration targets or can find the least possible project duration 


\section{Deleris, Bagchi, Kapoor, Katircioglu, Lam and Buckley}

for a given budget. It can also estimate the probability of achieving duration or budget targets, etc.

During project execution, after learning how the risk factors are affecting the activity durations, the algorithm can predict the remaining project cost and time to completion, assuming no changes in resources allocation (i.e., the optimization aspect of the algorithm is turned off). Such updates can be done regularly and can be valuable in taking risk mitigation actions. Coupled with such actions, the optimization aspect of the algorithm can be invoked to reoptimize resource scenario selection in order to bring a delayed project back on schedule with minimal possible cost.

We built a simulation environment to test the APRM analytics on an actual transition project. The first step in the simulation was to optimize the original project plan after incorporating risks and resource scenarios. The system was configured to minimize expected project cost subject to the expected project duration not exceeding a target. Based on the risks and resource scenario alternatives provided in the plan, the system projected an $11 \%$ reduction in project duration associated with a $9 \%$ project cost reduction. Assuming an unconstrained supply of resources, the resulting recommendations were recorded back in the project plan and effected immediately.

\subsection{Implementation Details}

In this section we describe the APRM simulation environment. We built a discrete event simulation model for the project plan using IBM's Websphere Business Modeler. We did the implementation of the BBN module using Java and the project planning optimizer using $\mathrm{C}++$. We developed a Service Modeling Object Library (SMOL) which comprises a variety of UML meta-models that capture the project plan resource scenarios, risks, roles, skills and costs. These were all integrated using SmartSCOR (Dong et al 2006), which is an IBM Research asset that provides a comprehensive framework for supply chain simulation and optimization.

Using a timer node in the simulation model, we call upon the BBN module and project planning optimizer providing as input the actual times of the completed activities. The recommended resource scenarios from the project planning optimizer are dynamically updated back into the simulation model. This enables the APRM environment to simulate the project plan and determine useful insights into optimal project execution given cost and time objectives.

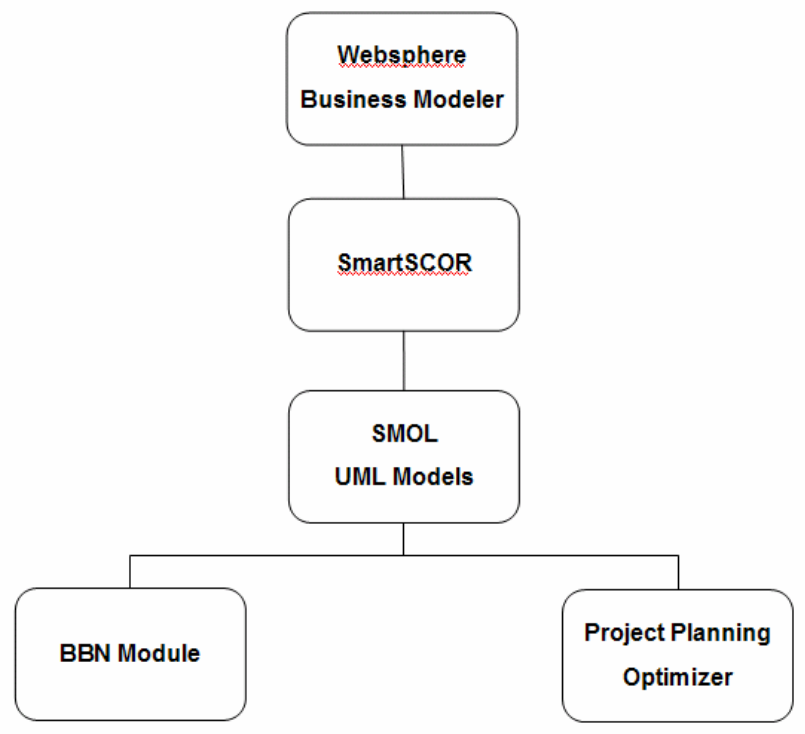

Figure 2: Simulation Environment for APRM

\section{EXPERIMENTS DESCRIPTION AND RESULTS}

\subsection{Experiment Description}

Our objective is to assess the value-added by periodically re-planning (i.e. re-allocating resources based on the updated risk profile.) Thus, to estimate the benefits from the APRM method, we simulate the evolution of the project plan under three alternatives:

- A - Monthly: resource allocation occurs at the start of the project and then at the end of each calendar month.

- $\mathrm{B}-$ Baseline: resource allocation occurs only at the start of the project.

- $\mathrm{C}$ - Base Case (No Planning or Re-planning): for each activity, the resource scenario with the minimum unit resource cost is chosen, regardless of the project duration risk for on time completion.

The resource allocation heuristic seeks to reach target project duration with minimum possible project cost. The project that we consider in these experiments takes an average of 145 days to complete for the base case. For our heuristic, we explore two possible project duration targets: 120 days (mild target) and 90 days (stringent target). In order to account for the randomness that stems from the risk profile, we did multiple replications of our simulations under different risk realizations (Monte Carlo simulation). In particular, we did 12 replications for the mild target of 120 days and 10 replications for the stringent target of 90 days. 


\section{Deleris, Bagchi, Kapoor, Katircioglu, Lam and Buckley}

\subsection{Performance of APRM}

This section investigates the performance of monthly replanning and baseline planning against the base case. For that purpose, we report the distribution of time and cost reduction brought by each alternative compared to the base case value. Figure 3 displays the box plots of the distribution of project cost reduction and Figure 4 of project duration reduction. Each box plot is a visual summary of the probability distribution of a sample set. Five points are reported: The minimum value, the lower quartile ( 25 percentile), the median, the upper quartile (75\%) and the maximum value.

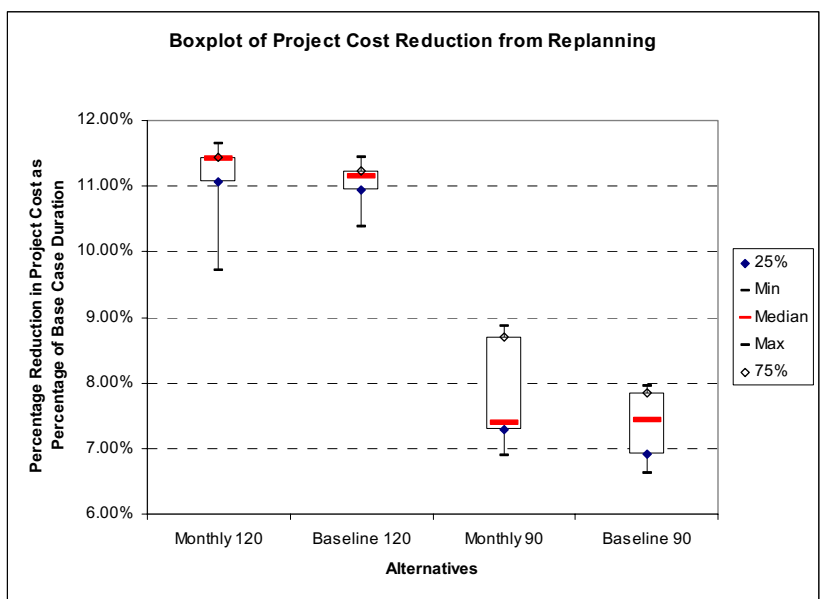

Figure 3: Reduction in project cost from using APRM. Comparison with base case values.

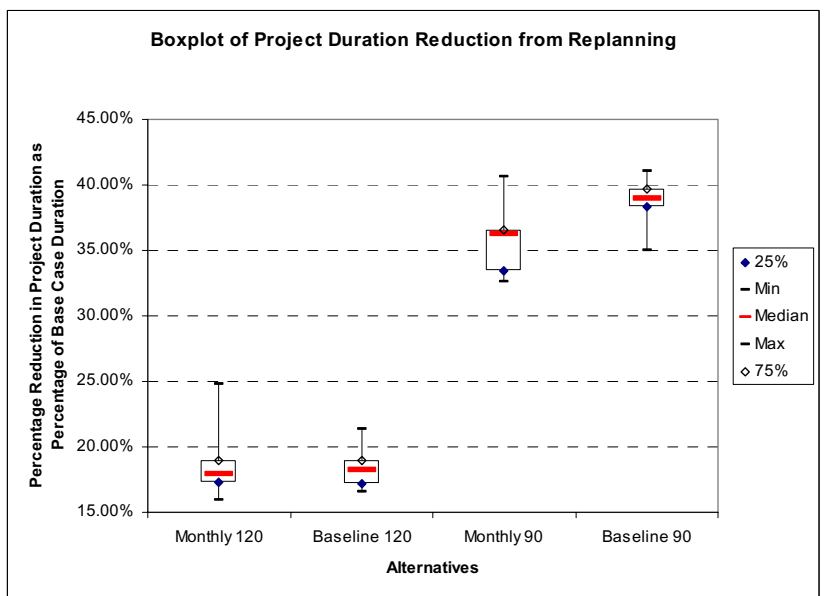

Figure 4: Reduction in project duration from using APRM. Comparison with base case values.

Overall using the APRM method, whether monthly or baseline, yields benefits in both project duration and project cost. For the mild target of 120 days, project duration is reduced by approximately $19 \%$ and project cost by about
$11 \%$. For the more stringent target, project duration is reduced by $35 \%$ to $40 \%$ on average and project cost by $7.5 \%$. As expected, the reduction in project cost is more important when the target is mild than when it is stringent.

Monthly re-planning and baseline planning appear to have similar performance levels, except under the stringent target where the project duration reduction is larger for baseline planning. Observe also that in all cases, monthly re-planning is more variable than baseline planning (lower minimum values, larger inter-quartile ranges, and higher maximum values).

\subsection{Comparison between Monthly Re-planning and Baseline Planning}

The previous results suggest that monthly re-planning and baseline planning are beneficial in terms of both cost and project duration. It is unclear, however, whether monthly re-planning provides additional benefits over baseline planning. Monthly re-planning is closest to the target duration $2 / 3$ of the cases and provides the lowest cost in $5 / 6$ of the cases. Table 1 shows all the combinations of closest time and least cost. Note however, that it is often the case that baseline planning yields lower project duration than monthly re-planning, but with a higher cost.

However, if we are not concerned about being closest to target project duration but rather about shortest project duration, the benefits from monthly re-planning are less obvious (see Table 2). While monthly re-planning is the less costly alternative in $5 / 6$ of the cases, it only yields shorter project durations in $1 / 4$ of the cases.

Table 1: Summary of the percentage of cases in which monthly re-planning performs better/worse than baseline planning in terms of cost and accuracy in meeting project target duration of 120 days.

\begin{tabular}{|l|l|c|c|}
\hline \multirow{2}{*}{} & & \multicolumn{2}{|c|}{$\begin{array}{r}\text { Closest to Project Duration } \\
\text { Target }\end{array}$} \\
\hline & & Monthly & Baseline \\
\hline \multirow{2}{*}{$\begin{array}{l}\text { Lowest } \\
\text { Cost }\end{array}$} & Monthly & $58.33 \%$ & $25.00 \%$ \\
\cline { 2 - 4 } & Baseline & $8.33 \%$ & $8.33 \%$ \\
\hline
\end{tabular}

Table 2: Summary of the percentage of cases in which monthly re-planning performs better/worse than baseline planning in terms of project cost and duration for 120 day project duration target.

\begin{tabular}{|l|l|c|c|}
\hline & & \multicolumn{2}{|c|}{ Shortest Project Duration } \\
\hline & & Monthly & Baseline \\
\hline \multirow{2}{*}{$\begin{array}{l}\text { Lowest } \\
\text { Cost }\end{array}$} & Monthly & $16.67 \%$ & $66.67 \%$ \\
\cline { 2 - 4 } & Baseline & $8.33 \%$ & $8.33 \%$ \\
\hline
\end{tabular}




\section{DISCUSSION \& CONCLUSION}

The experiments that we have undertaken clearly indicate that the APRM method can yield significant benefits in terms of project duration and total project cost. In fact, we observe that a project that takes about 145 days can be reduced to 120 days or even 90 days with a project cost reduction. Such an improvement is achieved by 1) accounting for the risks that affect the project 2) allocating best possible resources across activities to reduce the effect of those risks.

While monthly re-planning can reduce total cost compared to the baseline optimization, it often yields a longer absolute completion time. This may be due to the fact that the initial resource allocation from the baseline optimization is quite robust. Thus, periodic re-planning is not necessarily optimal as it could be overly reactive to random noise in the activity completion durations. Our intuition remains that such re-planning is still beneficial for extreme risk scenarios. In order to test the validity of this intuition, we selected a risk scenario that included a large number of delays. In that specific case, for the 90 days target, monthly re-planning yields a project duration of 93 days with a total project cost of $\$ 787,867$ while baseline planning yields a project duration of 96 days and a cost of $\$ 783,542$. Although more experiments should be conducted to explore this further, this result suggests that instead of re-planning periodically, we should consider dynamic re-planning when the project is predicted to be excessively delayed (according to some threshold).

Another situation where re-planning is expected to be useful is when resource allocation is needed across multiple projects. Due to cost concerns, resource utilization targets are generally kept at high levels. This makes the resource availability for projects very tight. An optimal resource allocation across different projects can improve resource utilization and project cost-time performance simultaneously. In a dynamic operational environment where new projects may appear and staffing needs may change unexpectedly, re-planning often can have merits. Re-planning can allow reallocating resources that are not critical for a project (i.e. resources assigned to activities not on the critical path) to critical activities of another project. This can enhance the effectiveness of resource use and improve overall performance of a project portfolio. A business process that allows such reallocation can also prevent project managers from using the critical resources for non-critical activities. In large companies where several projects exist at any point in time in different departments with minimal resource sharing, an integrated resource allocation process that allows resource sharing amongst these departments can bring significant cost and time improvements. The risk analysis and project optimization techniques we mentioned here can support better allocation de- cisions and therefore amplify the benefits of integrated resource allocation.

In this paper we presented our findings in a simulation study where we analyzed the effects of an adaptive project management method which uses analytics in risk estimation and project optimization. The risk estimation takes into account historical data and can learn during execution of a project in order to mitigate the estimated impact of risk factors on activity durations. The project optimization uses our APRM technique to minimize project cost subject to a project duration constraint by re-allocating resources during the course of a project. Our simulation study showed that optimal resource allocation that takes into account the impact of risk factors can significantly improve the cost and time performance of a project. Re-planning when projects are off target can also help under certain circumstances although they help only incrementally once resource optimization is done at the beginning of a project. We believe the potential benefits of optimal resource allocation with visibility to risk factors would be even higher in an environment where multiple projects compete for resources. Understanding the extent of the benefits in such an environment needs further studies, which we intend to conduct.

\section{ACKNOWLEDGMENTS}

The authors would like to acknowledge the useful feedback provided by Lianjun An, Young Lee, and Wei Wang during the course of the project.

\section{REFERENCES}

Cole, A. 1995. Runaway Projects - Cause and Effects. Software World (UK) 26(3), pp. 3-5.

Decision Systems Laboratory of the University of Pittsburgh. (http://dsl.sis.pitt.edu).

Deleris, L., Katircioglu, K., Bagchi, S., Kapoor, S., and Lam, D. 2007. Adaptive Project Risk Management. To appear in the proceedings of the 2007 IEEE/INFORMS International Conference on Service Operations and Logistics, and Informatics. Philadelphia, USA, August 27-29.

Dong, J., Ding, H., Ren, C., Wang. W. 2006. IBM SmartSCOR - A SCOR based supply chain transformation platform through simulation and optimization techniques. Proceedings of the 2006 Winter Simulation Conference.

Fan, C.-F. and Yu. Yuan-Chang. 2004. BBN-based Software Project Risk Management. J. Systems and Software 73(2), pp. 193 - 203.

Heckerman, D., Geiger, D. and Chickering, D. 1995. Learning Bayesian Networks: The Combination of Knowledge and Statistical Data. Machine Learning, 20(3) pp. 197-243. 
IBM Websphere Business Modeler (http://www306.ibm.com/software/integration/wbimodeler/)

Myers, J., Blackmond K. L., and Levitt, T. 1999. Learning Bayesian Networks from Incomplete Data with Stochastic Search Algorithms. Proceedings of the Genetic and Evolutionary Conference, Orlando, Florida.

Nasir, D., McCabe B. and Hartono, L. 2003. Evaluating Risk in Construction-Schedule Model (ERIC-S): Construction Schedule Risk Model. J. Construction Eng. and Management, 129(5), pp. 518-527.

Pearl, J. 1988. Probabilistic Reasoning in Intelligent Systems: Networks of Plausible Inference. San Francisco, CA: Morgan Kaufmann.

Taylor, H. 2005. The Move to Outsourced IT Projects: Key Risks from the Provider Perspective. Proceedings of the 2005 ACM SIGMIS CPR conference on Computer personnel research. Atlanta, Georgia, April 14-16.

Taylor, H. 2006. Critical Risks in Outsourced IT Projects: The Intractable and the Unforeseen. Comm. ACM 49(11), 75 .

\section{AUTHOR BIOGRAPHIES}

LEA A DELERIS is a post-doctoral researcher in the Mathematical Sciences department at IBM Research. She received her PhD in Management Science and Engineering from Stanford University. Her research interests lie with probabilistic risk analysis (applied widely for instance to supply chains, oil platform, or insurance firms) and decision analysis.

SUGATO BAGCHI is a Research Staff Member in the Mathematical Sciences department at the IBM T.J. Watson Research Center in Yorktown Heights, NY. His current research interests are in the application of simulation for operational decision-making in industries ranging from semiconductor to IT services.
SHUBIR KAPOOR is a senior engineer in the Mathematical Sciences department at the IBM Thomas J. Watson Research Center in Yorktown Heights. He has 10 years of significant work experience in the IT industry designing, architecting, programming complex algorithms and providing strategic and tactical design solutions, conducting logical analysis of technical problems and formulating mathematical models for solution of problems.

KAAN KATIRCIOGLU is a leading researcher and a consultant in supply chain management at IBM T J Watson Research Center. He has more than ten years of experience in the field of Operations Research, Management Science and Logistics. His expertise covers the areas of inventory optimization, distribution and manufacturing, e-business and supply chain management. Since he joined IBM's T.J. Watson Research Center in 1996, he has worked on several projects for various divisions of IBM and its customers. He has published several papers, made presentations at various conferences and received patents for his work. He is a member of INFORMS and IEEE.

RICHARD LAM is a Manager/Research Staff Member in the Mathematical Sciences department at the IBM T.J. Watson Research Center in Yorktown Heights, NY. He is investigating simulation models of service business processes using agent-based and system dynamics approaches.

STEVE BUCKLEY has been a Research Staff Member at the IBM Thomas J. Watson Research Center in Yorktown Heights, NY since 1987, and a manager at that facility since 1995. He currently manages the Analytic Models \& Architecture department in the Mathematical Sciences organization. He is an expert on supply chain management. His web page can be found via < http: //domino.research.ibm.com/comm/res earch_people.nsf/pages/buckley.index.ht ml> 